\title{
Initial Characterization of a Dedicated Breast PET/CT Scanner During Human Imaging
}

Spencer L. Bowen ${ }^{1}$, Yibao Wu ${ }^{1}$, Abhijit J. Chaudhari ${ }^{1}$, Lin Fu ${ }^{1}$, Nathan J. Packard ${ }^{2}$, George W. Burkett ${ }^{2}$, Kai Yang ${ }^{2}$, Karen K. Lindfors ${ }^{2}$, David K. Shelton ${ }^{2}$, Rosalie Hagge ${ }^{2}$, Alexander D. Borowsky ${ }^{3}$, Steve R. Martinez ${ }^{4}$, Jinyi Qi $^{1}$, John M. Boone ${ }^{2}$, Simon R. Cherry ${ }^{1}$, and Ramsey D. Badawi ${ }^{2}$

${ }^{I}$ Department of Biomedical Engineering, UC Davis, Davis, California; ${ }^{2}$ Department of Radiology, UC Davis Medical Center, Sacramento, California; ${ }^{3}$ Department of Pathology, UC Davis Medical Center, Sacramento, California; and ${ }^{4}$ Division of Surgical Oncology, UC Davis Cancer Center, Sacramento, California

We have constructed a dedicated breast PET/CT scanner capable of high-resolution functional and anatomic imaging. Here, we present an initial characterization of scanner performance during patient imaging. Methods: The system consisted of a lutetium oxyorthosilicate-based dual-planar head PET camera (crystal size, $3 \times 3 \times 20 \mathrm{~mm}$ ) and 768-slice cone-beam CT. The position of the PET heads (separation and height) could be adjusted for varying breast dimensions. For scanning, the patient lay prone on a specialized bed and inserted a single pendent breast through an aperture in the table top. Compression of the breast as used in mammography is not required. PET and CT systems rotate in the coronal plane underneath the patient sequentially to collect fully tomographic datasets. PET images were reconstructed with the fully 3-dimensional maximum a posteriori method, and CT images were reconstructed with the Feldkamp algorithm, then spatially registered and fused for display. Phantom scans were obtained to assess the registration accuracy between PET and CT images and the influence of PET electronics and activity on CT image quality. We imaged 4 women with mammographic findings highly suggestive of breast cancer (breast imaging reporting and data system, category 5) in an ongoing clinical trial. Patients were injected with ${ }^{18} \mathrm{~F}-\mathrm{FDG}$ and imaged for 12.5 min per breast. From patient data, noise-equivalent counting rates and the singles-to-trues ratio (a surrogate for the randoms fraction) were calculated. Results: The average registration error between PET and CT images was $0.18 \mathrm{~mm}$. PET electronics and activity did not significantly affect CT image quality. For the patient trial, biopsy-confirmed cancers were visualized on dedicated breast PET/CT on all patient scans, including the detection of ductal carcinoma in situ in 1 case. The singles-to-trues ratio was found to be inversely correlated with breast volume in the field of view, suggesting that larger breasts trend toward increased noiseequivalent counting rates for all other things equal. Conclusion: Scanning of the uncompressed breast with dedicated breast PET/CT can accurately visualize suspected lesions in 3 dimensions.

Key Words: breast cancer; PET/CT; positron emission mammography; ${ }^{18} \mathrm{~F}-\mathrm{FDG}$; dedicated

J Nucl Med 2009; 50:1401-1408

DOI: 10.2967/jnumed.109.064428

Received Mar. 17, 2009; revision accepted May 11, 2009.

For correspondence or reprints contact: Spencer L. Bowen, Department of Biomedical Engineering, University of California, One Shields Ave., Davis, CA 95616.

E-mail: slbowen@ucdavis.edu

COPYRIGHT ๑ 2009 by the Society of Nuclear Medicine, Inc.
W hole-body (WB) ${ }^{18}$ F-FDG PET has clinical utility in breast cancer staging, restaging, and therapy response assessment. A study by Rousseau et al. (1) found that WB PET could identify tumors with pathologic response after a single course of neoadjuvant chemotherapy (sensitivity, $61 \%$; specificity, 96\%), whereas mammography had limited accuracy (sensitivity, $31 \%$; specificity, 56\%), even after 6 courses of treatment. WB PET has been shown to have a high accuracy for detecting distant metastasis. Mahner et al. (2) measured a sensitivity and specificity for metastatic disease of $87 \%$ and $83 \%$, respectively, for WB PET, versus $43 \%$ and $98 \%$, respectively, for combined results from chest radiography, abdominal ultrasound, and bone scintigraphy.

The combination of WB PET with CT in a single platform (PET/CT) has been shown to have increased utility over either PET or CT alone for several oncologic imaging tasks (3). The CT component allows for the creation of fused images, showing the location of ${ }^{18}$ F-FDG uptake on an anatomic background, and allows the use of the low-noise radiographic scans for attenuation and scatter corrections. WB PET/CT, compared with either PET or CT alone, may also improve diagnostic confidence for breast cancers (4), especially for recurrent disease (5). With both WB PET and $\mathrm{PET} / \mathrm{CT}$, however, detection and quantification performance in breast cancer is significantly reduced when lesions are small $\left(<1 \mathrm{~cm}\right.$ diameter) or have low ${ }^{18} \mathrm{~F}$-FDG uptake with respect to the background $(6,7)$. The limited spatial resolution of WB PET, approximately $7 \mathrm{~mm}$ in full width at half maximum, and low coincidence photon detection sensitivity (photon sensitivity) for the breast are the main reasons for this performance loss.

Various groups are working on dedicated breast PET scanners, with the design emphasis on higher spatial resolution and photon sensitivity than are obtainable with WB PET. Potential breast PET applications include local staging, surgical planning, therapy response assessment, and residual or recurrent disease detection. Dedicated breast scanners can be generalized into 2 groups. Positron emission mammography (PEM) systems use 2 planar $(8-10)$ or 
curved (11) detector heads and image the breast under mild compression, with limited-angle tomography. In a clinical trial of 94 women scanned on a commercial PEM device, with mammographic and clinical examination findings available to the study readers, PEM, compared with WB PET, showed a significantly improved sensitivity for subcentimeter lesions (12). Although in-plane PEM resolution is relatively high $(2-3 \mathrm{~mm})$, out-of-plane resolution is degraded because of incomplete angular coverage (13). Scanners in the second group, termed bPET, acquire fully tomographic images of the breast by rotating 2 or more planar heads $(14,15)$ or by completely encircling the breast with detectors (16-18). bPET systems produce images with isotropic spatial resolution (thereby potentially limiting the superimposition of structures, compared with PEM). The performance of a bPET system for in vivo imaging, however, has not been reported to date.

We have constructed a combined, dedicated bPET/CT scanner. The goal of this study was to assess the performance of this system during phantom measurements and patient scanning.

\section{MATERIALS AND METHODS}

\section{System Description}

The UC Davis dedicated breast scanner, herein referred to as DbPET/CT, consisted of a dual-head PET camera and cone-beam CT integrated into a single gantry (Fig. 1A). For imaging, the patient was positioned prone with a single pendent breast hanging into the field of view (FOV) of the scanner. In contrast to WB PET/CT, the DbPET/CT transaxial FOV is parallel and the axial FOV perpendicular to the coronal plane (Fig. 1A).

The CT component was composed of a CsI flat-panel detector (PaxScan 4030CB; Varian Medical Systems), a tungsten target radiograph tube (Comet AG), and a custom-made rotational gantry (Fig. 1A). Characteristics of the system are given in Table 1. Performance results of an earlier breast CT prototype with similar characteristics (19) and results from a patient trial (20) have been reported.

The PET component uses lutetium oxyorthosilicate-based detector modules arranged into 2 square flat-panel heads. Table 2 summarizes the key parameters of the camera. A detailed description of the scanner and its basic performance measurements have been reported (15). Briefly, each single-ended readout detector module was composed of a crystal array coupled to a positionsensitive photomultiplier tube (R5900-C8; Hamamatsu Photonics) via an optical fiber bundle (21). For electronics, single-event triggers are subject to a 12 -ns coincidence window $(2 \tau)$ with no offset for prompts or a 32-ns delay for delayed coincidences.

PET heads are mounted on a custom-built gantry that allows 3 degrees of freedom and shielding (Fig. 1B). Detector rotation around the center FOV, separation distance, and height are all adjusted by individual computer-controlled drives (Fig. 1B). Photon sensitivity can be maximized for a given breast by minimizing the detector separation distance. The detector height adjustment allows for the distance between the top of the PET heads and patient chest wall to be minimized while still allowing space for rotational clearance. To aid in patient positioning, a hand-controller allows independent control of all drives. For
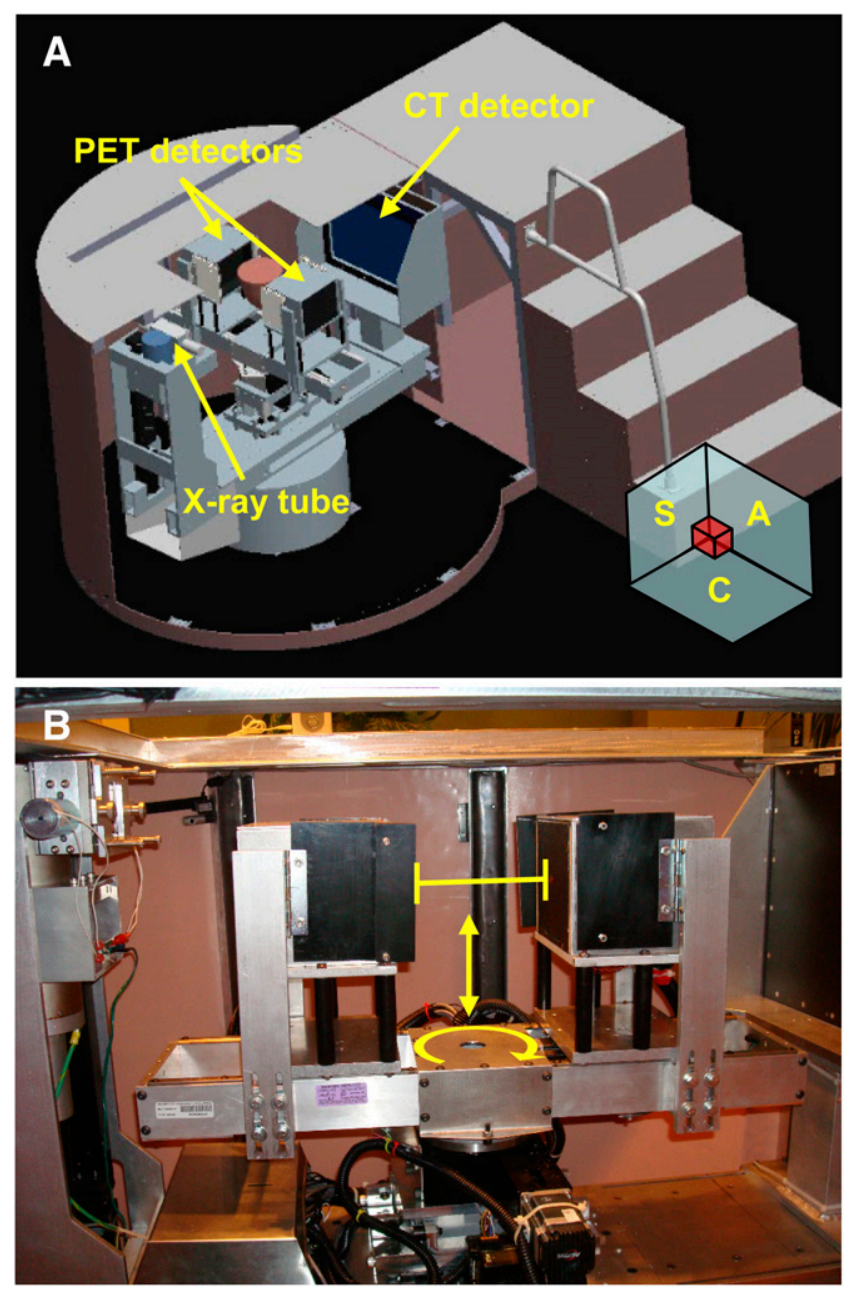

FIGURE 1. (A) Schematic depicting DbPET/CT. Object between PET detectors shows approximate position of patient's breast during scanning. Orientation of positioned patient's coronal $(C)$, sagittal $(S)$, and axial plane $(A)$ are depicted in bottom-right-hand corner. (B) PET gantry allows for control of detector height (vertical arrow), separation distance (horizontal line with end markers), and rotation (curved arrow).

shielding from x-rays, 3-mm-thick lead plates cover the front of PET heads during CT and line 3 sides (excluding the back, front, and bottom) of each PET head. The total distance between the crystal arrays and top of the PET head is $0.9 \mathrm{~cm}$.

\section{Patient Bed and Positioning Aids}

Placement of the patient's breast in the scanner FOV is handled by the patient bed and breast-positioning system. With the custom bed, a sloped steel table top allows the patient to comfortably bend at the hips, and a carbon fiber support and Naugahyde cover (Uniroyal Engineered Products LLC) surrounding the aperture in the table top permit the patient to sink under her own weight. The combination of these elements allows the anterior aspect of the patient to be positioned significantly farther into the top of the axial FOV of the scanner than if a flat and rigid table were used. Because the transaxial PET FOV $(11.9 \mathrm{~cm})$ is less than the average breast diameter $(14.0 \mathrm{~cm})(22)$, a breast-positioning system (composed of 


\section{TABLE 1. CT System Characteristics}

Component Characteristic

Radiograph Focal spot: water-cooled $\mathrm{W}$ anode, beryllium tube window, $0.4 \times 0.4 \mathrm{~mm}$, and $0.3-\mathrm{mm}$ copper-added filtration

$1,000 \mathrm{~W}$ (i.e., maximum amperage at $80 \mathrm{kVp}=12.5 \mathrm{~mA}$ )

Detector Material: indirect detection (CsI scintillator) thin film transistor

Active area: $40 \times 30 \mathrm{~cm}$

Native pixel matrix: 2,048 $\times 1,536$

Native pixel size: $0.194 \times 0.194 \mathrm{~mm}$

$2 \times 2$ pixel binned matrix: $1,024 \times 768$

Frame rate: 30 frames/s at $2 \times 2$ binning

Data are adapted from Boone et al. (37)

a clear polycarbonate cylinder with ports for technician access) is used to center the patient's breast.

\section{Acquisition}

A CT acquisition preceded a PET acquisition unless otherwise noted. For CT, 500 projections were taken over $16.6 \mathrm{~s}$, with a continuous rotation over $360^{\circ}$. On the basis of the percentage of glandularity and size of a given breast (23), tube current was adjusted to deliver the same dose as 2-view mammography (range for patient imaging, 2.5-7.3 mA), whereas tube voltage was fixed at $80 \mathrm{kVp}$. PET heads were positioned and then rotated in a step-andshoot motion (40 steps) over $180^{\circ}$. Acquisition time for the PET was user-defined but was typically approximately $10 \mathrm{~min}$ per breast.

\section{Data Processing and Reconstruction}

For the PET component, coincidence data were passed through a 350-650 keV energy window (crystal-by-crystal basis) before conversion to list mode. Data were reconstructed with a fully 3-dimensional maximum a posteriori (MAP) (24) based algorithm into an image of $108 \times 108 \times 36$ voxels of dimension $1.1 \times 1.1 \times 3.3 \mathrm{~mm}$ (transaxial sampling, $1.1 \mathrm{~mm}$ ). For this study, reconstructed data were corrected for center-of-rotation offset and geometric efficiency factors. Randoms subtraction, scatter, attenuation, and dead-time corrections were not implemented.

CT data were reconstructed with the Feldkamp (25) algorithm and normalized to Hounsfield units (HU). An image volume is composed of a number $(n)$ of coronal images with a voxel matrix of $512 \times 512 \times n$, with $n$ set to contain a given breast length. For patient imaging, the voxel dimensions range from 0.2 to $0.4 \mathrm{~mm}$ transaxially and from 0.2 to $0.3 \mathrm{~mm}$ axially.

\begin{tabular}{|c|c|}
\hline Parameter & Value \\
\hline Crystal size (mm) & $3 \times 3 \times 20$ \\
\hline Crystal array & $81(9 \times 9)$ \\
\hline Crystal pitch (mm) & 3.3 \\
\hline No. of detector blocks & $16(4 \times 4)$ \\
\hline FOV $(\mathrm{cm})$ & 11.9 (axial + transaxial) \\
\hline Lines of response & $1,296^{2}$ \\
\hline
\end{tabular}

Fused images were created by registering, with an affine transform and trilinear interpolation, the PET data to CT image space via RView (26). Image volumes from the 2 modalities are displayed using MRIcroN (27) with a gray scale for CT and X_hot color scale for PET.

\section{Registration Accuracy}

Registration accuracy between the PET and the CT components was assessed using a phantom containing 4 refillable spheres with an inner diameter of $5 \mathrm{~mm}$ (Data Spectrum Corp.) arranged at several heights and filled with ${ }^{18} \mathrm{~F}-\mathrm{FDG}$ and iodine contrast. The phantom was fixed at the center of the transaxial FOV and imaged once by CT. To examine registration accuracy as a function of detector position, PET (acquisition time, $12.5 \mathrm{~min}$; head separation, $262 \mathrm{~mm}$ ) was performed at 6 detector heights at intervals of $13.4 \mathrm{~mm}$. PET images from the lowest $(0 \mathrm{~mm})$ and highest heights were manually registered with an affine transform to the CT images using RView (26). Values in the transformation matrices, describing the registration, between the 2 extreme heights were assumed to vary linearly as a function of detector height and were described by parametric equations. Repositioning accuracy of the gantry was assessed by imaging the 4-sphere phantom a total of 7 times, parking and then repositioning the scanner between acquisitions. PET images for the single height were registered to CT images using the parametric equations calculated in the detector position study. Error in registration was quantified by computing the Euclidian distance between sphere center of masses $(\mathrm{CM})$ in the PET and CT domains.

\section{Influence of PET on CT}

The effect of the PET electronics or activity on CT image quality was quantified. The influence of the CT component on PET has been reported previously (15). A plastic refillable jar (inner diameter, $14 \mathrm{~cm}$ ) and $70-\mu \mathrm{m}$-thick nickel-chromium wire arranged perpendicularly to the transaxial FOV of the scanner were imaged by CT in 3 different configurations in the following order: a jar filled with water only and with the PET high-voltage (HV) off, jar filled with water only and with the PET HV on, and jar filled with $259 \mathrm{MBq}$ of ${ }^{18} \mathrm{~F}-\mathrm{FDG}$, at the start of imaging, and with PET HV on. From the reconstructed CT images, the modulation transfer function (MTF) was estimated from the wire as previously described (19). To estimate image uniformity, individual circular regions of interest (diameter, $12 \mathrm{~cm}$ ) were drawn on coronal image slices centered on the cylinder, for slices spanning the axial FOV of the scanner. The mean and SD of voxel HU for each region of interest were computed.

\section{Patient Trial}

A clinical trial is currently being conducted with DbPET/CT involving women highly suspected of having breast cancer (breast imaging reporting and data system, category 5) as determined through mammography (28). Currently, we have imaged a total of 7 breasts from 4 patients ( 1 patient underwent a prior mastectomy). This and related protocols have been approved by the UC Davis Medical Center institutional review board and require written consent from the patient. Eligible patients were age 3580 y (age range, 49-70 y), had not had a recent breast biopsy, and were not pregnant or diabetic. Before the injection with ${ }^{18} \mathrm{~F}-\mathrm{FDG}$ (range, 174-477 MBq), patients fasted for more than $4 \mathrm{~h}$ and were checked with a finger-stick test to ensure normal blood glucose levels $(<200 \mathrm{mg} / \mathrm{dL})$. Patients were asked to void their bladder before being positioned on the scanner with the affected breast in 
the FOV first, unless otherwise noted. A CT scan was obtained, with the patient coached to perform end-expiration breathholding. The technician then used the hand-controller to position the PET heads as close as possible to the patient's chest wall. The patient was advised to breathe normally and was scanned for 12.5 min by PET (average uptake time, $81 \mathrm{~min}$; range, 73-89 $\mathrm{min}$ ). Patients were then repositioned for unaffected breast imaging. All patient images presented were windowed between -450 and 250 $\mathrm{HU}$ for $\mathrm{CT}$ and between $0 \%$ and $95 \%$ maximum image intensity for PET, unless otherwise noted. Additionally, dedicated CT images represent an average of 3 slices in the plane displayed.

For 1 patient, a modified protocol was used for DbPET/CT, with an intravenous CT contrast agent. Scanning proceeded as follows: imaging of the unaffected breast as detailed above, scanning of the affected breast with PET, and scanning with $\mathrm{CT}$ before and at $35 \mathrm{~s}$ after injection of $100 \mathrm{~mL}$ of iodixanol (320 $\mathrm{mgI} / \mathrm{mL}$ ) (Visipaque 320; GE Healthcare) with a power injector (Mark V Plus; Medrad). A contrast-subtraction image was produced by subtracting pre- and post-contrast-enhanced CT scans rigidly registered with RView (26).

Patients underwent additional imaging tests as part of their standard work-up. The suggestion of multifocal or multicentric disease or inconclusive findings on mammography (breast imaging reporting and data system, category 0) prompted 3 patients to undergo bilateral dynamic contrast-enhanced MRI (DCE-MRI). One patient with the suggestion of distant spread was scanned in the prone position on WB PET/CT (Discovery ST; GE Healthcare) (acquisition time, $5 \mathrm{~min} / \mathrm{bed}$ position), with images reconstructed by the manufacturer's software as follows: PET, ordered-subset expectation maximization ( 2 iterations, 30 subsets), with voxels of $5.1 \times 5.1 \times 3.3 \mathrm{~mm}$, and CT, with voxels of $1.0 \times 1.0 \times 3.7 \mathrm{~mm}$.

An effort was made to spatially register tomographic images with histologic findings. A mastectomy sample was cut by hand in sagittal slices (slice thickness, $\approx 5 \mathrm{~mm}$ ), and photographic images were obtained for each slice. Histology was performed at several locations on a slice with suspected lesions. For comparison, DbPET/CT, DCE-MRI, and WB PET/CT sagittal slices were selected qualitatively on the basis of the similarity of fibroglandular structure with the tissue section. Sagittal sections were aligned unaltered (i.e., without corrections for soft-tissue deformation or rigid rotations of the breast).

For DbPET/CT image interpretation, 1 board-certified radiologist specializing in breast imaging reviewed only the CT images, and a second radiologist with expertise in nuclear medicine reviewed the fused image sets (the CT image was used only as an anatomic reference, and a final interpretation was made on the basis of the PET image). Readers had access to all prior breast examinations and images from mammography, DCE-MRI, and WB PET, if available, including the interpretation of the dedicated CT images in the case of the fused image reader. On the basis of the qualitative metrics, each reader determined if suspected lesions (positive) were present on DbPET/CT images and, if so, correlated these findings with histopathology.

\section{Counting Rate Estimations from Patient Scans}

Noise-equivalent counting rates (NECRs) were estimated from patient scans (29). To only include randoms (estimated from delayed coincidences) and scatters with lines of response (between crystals $i$ and $j$ ) passing through breast tissue at a given projection angle $(\phi)$, a binary histogram mask $\left(\mathrm{m}_{\mathrm{ij \phi}}\right)$ defining the interior of each breast was generated from patient PET images (30). The scatter fraction $\left(\mathrm{sf}_{\phi}\right)$ was estimated using the Monte Carlo simulation software GATE (31). A digital phantom of each patient's breast was composed of both an activity and an attenuation map estimated from the original patient PET images. Activity from outside the FOV (e.g., patient torso) was not included because this was not expected to significantly contribute to the total scatter fraction (32).

Integral counts for trues $\left(\mathrm{T}_{\phi}\right)$, randoms $\left(\mathrm{R}_{\phi}\right)$, and prompts $\left(\mathrm{P}_{\phi}\right)$ and NECR values were calculated as follows:

$$
\begin{array}{cc}
\mathrm{T}_{\phi}=\left(1-\mathrm{sf}_{\phi}\right) \sum_{\mathrm{i}} \sum_{\mathrm{j}}\left(\mathrm{p}_{\mathrm{ij} \phi}-\mathrm{r}_{\mathrm{ij} \phi}\right) \mathrm{m}_{\mathrm{ij} \phi}, & \text { Eq. } 1 \\
\mathrm{R}_{\phi}=\sum_{\mathrm{i}} \sum_{\mathrm{j}} \mathrm{r}_{\mathrm{ij} \phi} \mathrm{m}_{\mathrm{ij} \phi}, & \text { Eq. } 2 \\
\mathrm{P}_{\phi}=\sum_{\mathrm{i}} \sum_{\mathrm{j}} \mathrm{p}_{\mathrm{ij \phi}} \mathrm{m}_{\mathrm{ij} \phi}, & \text { Eq. } 3 \\
\mathrm{NECR}=\frac{\frac{1}{\Delta \mathrm{t}}\left(\sum_{\phi} \mathrm{T}_{\phi}\right)^{2}}{\sum_{\phi} \mathrm{P}_{\phi}+(\mathrm{k}-1) \sum_{\phi} \mathrm{R}_{\phi}}, & \text { Eq. } 4
\end{array}
$$

where $p_{i j \phi}$ and $r_{i j \phi}$ are prompts and randoms counts, and $\Delta t$ is the total acquisition time. In Equation $4, \mathrm{k}=2$ or $\mathrm{k}=1$ for direct or variance-reduced randoms subtraction, respectively. For each patient, the injection dose of ${ }^{18}$ F-FDG was normalized to a value that would give the same initial total activity in the patient after a 60-min uptake, as the actual average activity present during the scan as previously described (30).

To assess the relative contribution of randoms as a function of breast volume, the energy-qualified singles-to-trues ratio (STR) was computed for patient scans. The STR is a surrogate for the randoms fraction, with the advantage that it does not depend on activity in the FOV. As energy was not recorded for singles, events falling in the $350-650 \mathrm{keV}$ window were estimated by scaling recorded singles by the square root of the ratio of windowed-tononwindowed randoms. In addition, both singles and trues were dead-time-corrected to account for differences in the system counting rate response.

\section{RESULTS}

\section{Registration Accuracy}

In examining registration accuracy as a function of detector position, we determined that the largest Euclidian distance between the $\mathrm{CM}$ of a single sphere $(0.34 \mathrm{~mm})$ occurs at a detector height of $67.2 \mathrm{~mm}$. Average error for all 4 spheres over all heights is $0.16 \pm 0.08 \mathrm{~mm}$. CM error does not significantly increase from the minimum average error $(0.14$ $\mathrm{mm}$ ) as a function of vertical offset. For the repositioning study, the average error for all 4 spheres across all repositions is $0.20 \pm 0.10 \mathrm{~mm}$. Only registration error at the fifth reposition $(0.43 \pm 0.17)$ is significantly greater $(P=0.004)$ than the total average. 

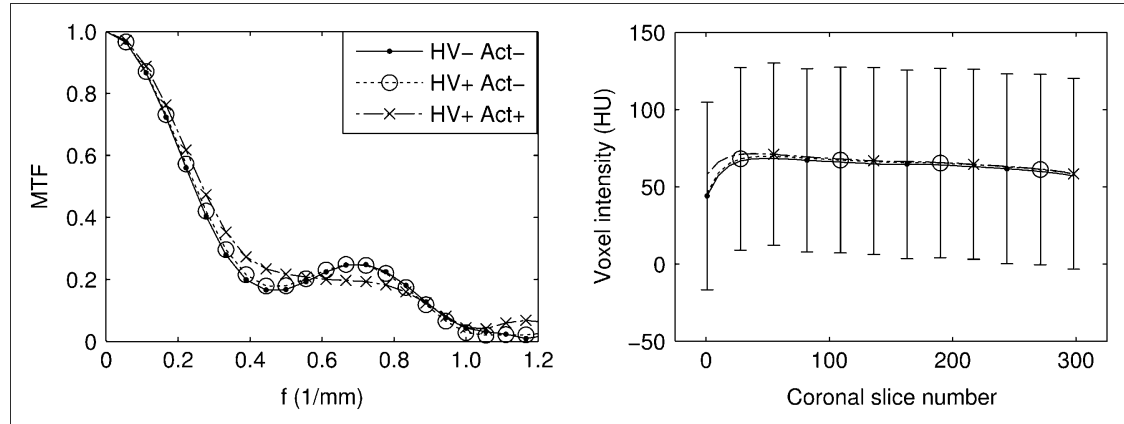

FIGURE 2. Influence of PET electronics and activity on CT image quality for $\mathrm{HV}$ off and no activity in FOV (HV - Act-), PET HV on and no activity (HV+ Act-), and PET HV on and activity present $(\mathrm{HV}+$ Act + ). MTF vs. line pair frequency (f; left). Image uniformity (mean and SD bars) as function of CT coronal slice number (lower magnitude is more posterior) (right). SD bars are representative of typical values and are staggered between imaging scenarios for clarity.

\section{Influence of PET on CT}

Figure 2 (left) shows the influence of the PET component on the MTF of the CT. The MTF curve computed with only PET HV on (HV+ activity [Act]-) does not differ significantly from the water-only scan (HV-Act - ). The MTF with $\mathrm{HV}$ on and activity $(\mathrm{HV}+\mathrm{Act}+)$ differs by at most 0.074 from the other imaging scenarios (frequency, $0.33 \mathrm{~mm}^{-1}$ ). The difference is likely insignificant and may be attributed to the subtle variation of image artifacts induced by slight motion of the uniform cylinder during activity filling.

Figure 2 (right) shows how CT image uniformity is affected by the PET component. Differences in mean HU between the water-only scan and acquisitions with PET HV $(\mathrm{HV}+\mathrm{Act}-)$ and activity $(\mathrm{HV}+\mathrm{Act}+)$ are not significantly greater than the interscan HU fluctuations measured on the CT component alone.

\section{Patient Trial}

Table 3 summarizes the radiologic interpretation of DbPET/CT images from the patient trial. For patient 2, an earlier iteration of the patient bed limited the volume of breast tissue visible on CT such that the invasive lobular carcinoma was above the top of the CT axial FOV. In patient 3, the CT component was able to visualize calcifications representative of ductal carcinoma in situ (DCIS), but the PET images were not interpretable because of inaccurate registration, likely because of patient motion.

Figure 3 shows DbPET/CT images for patient 1's affected breast. The 49 -y-old patient presented with a palpable 23-mm irregular focal mass at the 8 o'clock position, as seen on mammography. The axial fused image (Fig. 3A) shows 3 separate areas of focal uptake visible on PET overlying fibroglandular tissue as visualized by CT.

Figure 4A shows a tissue section excised from the mastectomy sample of patient 1 . Histology results in Figure
4B obtained at several locations on the tissue section show a band of DCIS superior to a benign region of fibroglandular tissue. Figure 4C shows a fused sagittal DbPET/CT image corresponding to the tissue section. Areas of increased uptake on PET overlay malignancies (Fig. 4C, boxes i-iii), and a region with uptake not significantly above background (Fig. 4C, box iv) overlays benign tissue. No indications of DCIS were visible on the CT images alone (Table 3). Figure 4D shows a sagittal image from a WB PET/CT acquisition (tube voltage, $140 \mathrm{kVp}$; injection activity, $466 \mathrm{MBq}$; uptake time, $76 \mathrm{~min}$ ) obtained $29 \mathrm{~d}$ after imaging with DbPET/CT. The fused image from DbPET/CT (Fig. 4C), compared with WB PET/CT (Fig. 4D), shows qualitatively improved resolution for both the PET and the CT components. Regions of increased contrast visible on DbPET/CT (Fig. 4C) correlated well with those seen on DCE-MRI (Fig. 4E).

Figures 5A and 5B show $\mathrm{CT}$ and fused sagittal images, respectively, of the affected breast of patient 4 . The 66-yold patient presented with a $20-\mathrm{mm}$ spiculated mass at the 10 o'clock (posterior third) position as seen on mammography. The fused image (Fig. 5B) shows 2 areas of focal uptake anterior and posterior on PET, determined by biopsy to be multifocal cancer (Table 3). The proximity of the top of the PET axial FOV with respect to patient 4's pectoralis muscles is also visualized in Figure 5B. Figure 5C shows the CT contrast subtraction image.

\section{NECR from Patient Scans}

Table 4 shows NECR values estimated from patient scans. Average NECR $(\mathrm{k}=1)$ was 511 counts per second (cps) (range, 107-1,474 cps), with the largest NECR for the affected breast of patient 1 (breast number, 1A) (trues, 2,575 cps; randoms, $819 \mathrm{cps}$ ). The results show the scatter fraction to be significantly correlated with breast volume in the FOV $\left(R^{2}, 0.92\right.$ for linear fit). In addition, subtraction with a

\section{TABLE 3. Radiologic Interpretation for DbPET/CT-Affected Breast Images}

\begin{tabular}{|c|c|c|c|c|c|}
\hline \multirow[b]{3}{*}{ Cancer type } & \multicolumn{5}{|c|}{ Patient no. } \\
\hline & \multicolumn{2}{|l|}{$\begin{array}{r}1 \\
\end{array}$} & \multirow{2}{*}{$\frac{2}{\text { Invasive lobular }}$} & \multirow{2}{*}{$\frac{3}{\mathrm{DCIS}}$} & \multirow{2}{*}{$\frac{4}{\frac{4}{\text { Invasive ductal }}}$} \\
\hline & Invasive mammary & DCIS & & & \\
\hline CT-positive & Yes & No & No & Yes & Yes \\
\hline PET-positive & Yes & Yes & Yes & No & Yes \\
\hline
\end{tabular}


FIGURE 3. Axial (A) and coronal (B) DbPET/CT images from affected breast of patient 1. CT, PET, and fused images are presented from left to right.
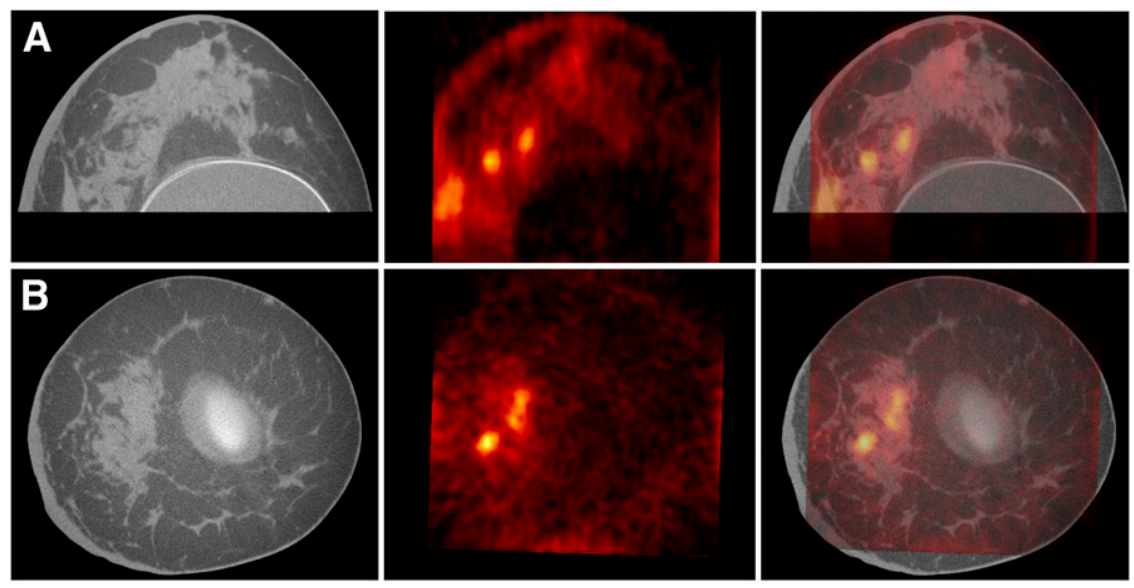

variance-reduced randoms estimate $(\mathrm{k}=1)$ increases NECR by up to $75 \%$ (average for all breasts, $41.3 \%$ ), compared with direct subtraction $(\mathrm{k}=2)$, for the breast imaged with the largest normalized injection activity (breast number, 3A). Figure 6 shows the STR plotted as a function of breast volume. The largest STR was for breast number 3A (ratio, 722), whereas the smallest was for breast number $1 \mathrm{~A}$ (ratio, 92).

\section{DISCUSSION}

Scanning of the uncompressed breast with DbPET/CT can produce fully 3-dimensional images that accurately show the size, extent, and location of biopsy-confirmed breast cancer. For patient 1, invasive carcinomas were visible adjacent to a breast implant (Fig. 3). Implants may reduce the sensitivity of mammography even with implant displacement views (33). In this same patient, features presenting on the functional and anatomic images from DbPET/CT correlated well with histologic results and gross anatomy, respectively (Fig. 4). The histologic correlation for patient 1 , along with the radiologic interpretation (Table 3) of patients 1, 2, and 4 (Fig. 5), suggests that PET images can be accurately registered to CT images during human imaging; however, in patient 3 registration appeared degraded because of patient motion. The use of a special- ized breathing protocol for breast imaging, improvements in the patient bed, and mild compression could all potentially reduce registration error.

For patient 1, a DbPET/CT scanner, compared with a commercial WB PET/CT scanner (Fig. 4D), demonstrated qualitatively improved visualization of DCIS (Fig. 4C). A patient trial with PEM (12) measured a sensitivity for DCIS (91\%) significantly higher than values typically reported for WB PET. A known limitation in our comparison was the method used for registering tomographic image slices (WB PET/CT, MRI, or DbPET/CT) to the tissue section. Sagittal slices were aligned unaltered on the basis of qualitative matching, resulting in visibly reduced spatial correlation. Other factors potentially biasing the intermodality comparison include differences in acquisition parameters, counting rates, reconstruction algorithms, and correction methods. Nevertheless, we believe that the increased resolution of the dedicated versus WB scanner for both the PET (average full width at half maximum for WB, $6.4 \mathrm{~mm}$; average full width at half maximum for dedicated, $3.7 \mathrm{~mm})(15,34)$ and the CT (average resolvable line pairs for WB, $0.7 \mathrm{~mm}^{-1}$; dedicated, $\left.1.1 \mathrm{~mm}^{-1}\right)(19,35)$ results in an appreciable improvement in lesion visualization for a patient who was scanned with typical clinical acquisition protocols.
FIGURE 4. (A) Sagittal tissue section excised from mastectomy sample of patient 1's affected breast with 4 areas (boxes) of histology performed. (B) Histology tissue slides with magnified regions (right, corresponding to black boxes) revealed DCIS alone (i-ii) or with intralymphatic invasion (iii, not shown) and benign tissue (iv). DbPET/CT (C), WB PET/CT (D), and DCE-MRI (E) sagittal image slices corresponding to tissue section (A). Boxes in DbPET/CT image (C) are at locations approximating those in tissue section (A). PET images ( $C$ and $D$ ) were windowed between $0 \%$ and $60 \%$ maximum image intensity.
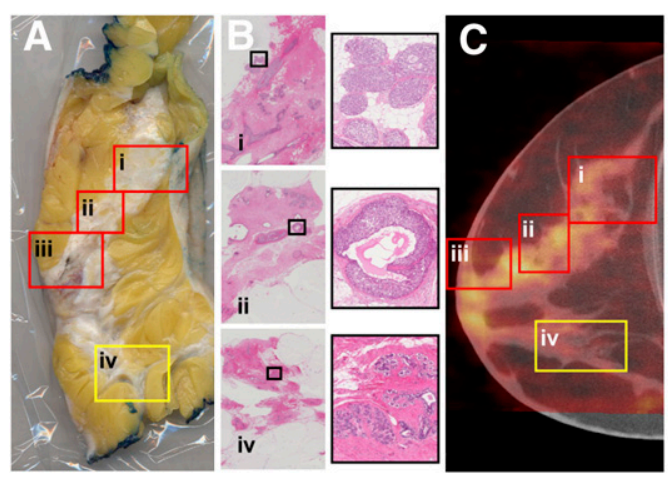

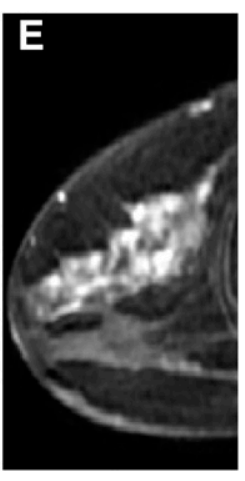




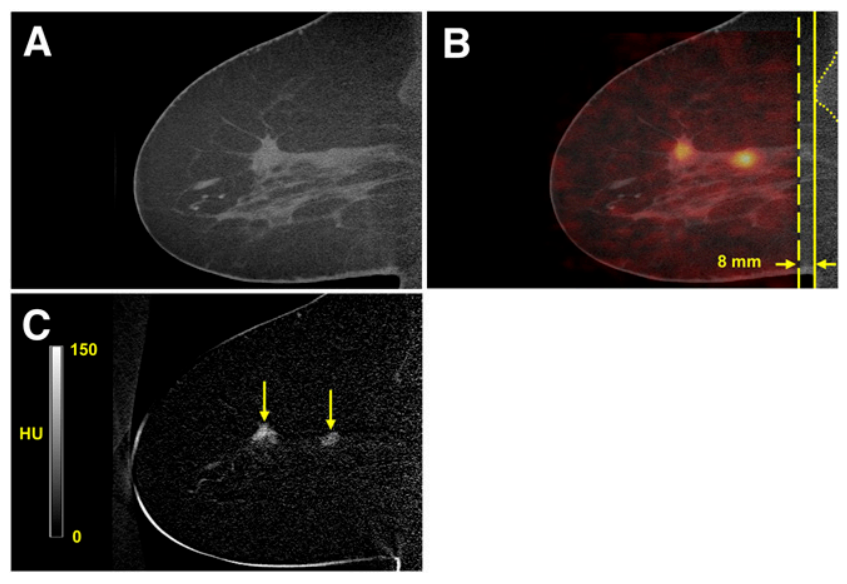

FIGURE 5. Pre-contrast-enhanced CT (A), fused PET/CT(B), and contrast subtraction sagittal DbPET/CT (C) images showing affected breast of patient 4. Two areas of focal uptake were seen on PET (B) and on contrast subtraction CT (C) (arrows). (B) Distance (opposing arrows) between top of PET axial FOV (dashed line) and anterior aspect (solid line) of pectoralis muscles (dotted line) is shown. (C) Contrast subtraction image is average of 7 slices and uses alternative windowing.

Besides providing anatomic reference, the CT component of DbPET/CT, compared with breast PET alone, increases the overall system functionality. In patient 4 , the combination of increased radiograph density with ${ }^{18} \mathrm{~F}-\mathrm{FDG}$ uptake or iodine contrast (Fig. 5) accurately localized a suspected lesion that was originally occult on screening mammography. Although iodinated contrast and ${ }^{18}$ F-FDG have high spatial correlation in this case, the kinetics of the 2 tracers are regulated by independent physiologic processes (angiogenesis for iodinated contrast vs. glucose metabolic rate for ${ }^{18} \mathrm{~F}-\mathrm{FDG}$ ), such that differences in iodinated contrast and ${ }^{18} \mathrm{~F}-\mathrm{FDG}$ uptake could potentially improve reader confidence or quantitative measures for a given lesion. The CT component may also improve the utility of recently developed robotic biopsy devices $(14,20)$. Fused 3-dimensional DbPET/CT images would allow for accurate needle placement, and the CT, operating in low-dose fluoroscopy mode, could provide real-time needle guidance.
NECR values from patient scanning (Table 4) are influenced significantly by breast volume in the scanner FOV. In contrast to WB PET systems, the randoms fraction for the dedicated PET scanner, as estimated by the STR (Fig. 6), is inversely related to the volume of tissue in the FOV (30). This inverse relationship supports predictions that image noise for prone dedicated breast PET scanners may be significantly influenced by activity from outside the FOV (36). The large magnitude of singles flux from the brain, torso, or bladder dominates any increase in singles with breast volume. Because of the relatively small range of breast dimensions, compared with those of the torso, loss of trues from self-attenuation does not appear to play a significant role. Assuming relatively constant singles flux, the randoms fraction declines more rapidly than the scatter fraction increases as a function of breast volume, and all other things being equal, NECR is greater for larger breasts.

Some limitations exist for patient imaging with the current DbPET/CT. First, chest wall and breast axillary tail coverage of both modalities is restricted because of the geometric constraints inherent with prone imaging. With the current bed setup, the top of the axial FOV for the CT can be positioned closer to the chest wall than for the PET; a 20-mm difference was measured in 1 patient (Fig. 5B). The chest wall coverage limitations are likely worse for rotational systems; however, in 2 clinical imaging studies with PEM false-negatives were reported when lesions were above the scanner axial FOV $(8,12)$. Second, DbPET/CT, although supporting all necessary measurements for quantification, is not able to produce fully quantitative images at this time. Accurate quantification is likely to be important when using breast PET to monitor therapy response, and efforts to achieve such are ongoing.

\section{CONCLUSION}

We have assessed the clinical performance of a dedicated breast PET/CT scanner through phantom measurements and patient scanning. Preliminary clinical results demonstrate that dedicated tomographic scanning of the uncompressed breast can accurately visualize suspected lesions in 3 dimensions. More research is required to determine

\begin{tabular}{|c|c|c|c|c|c|c|c|}
\hline \multirow[b]{2}{*}{ Parameter } & \multicolumn{7}{|c|}{ Breast no.* } \\
\hline & $1 \mathrm{~A}$ & $1 U$ & $2 \mathrm{~A}$ & $2 U$ & $3 \mathrm{~A}$ & $4 \mathrm{~A}$ & $4 U$ \\
\hline Normalized injection activity (MBq) & 196 & 157 & 324 & 270 & 382 & 118 & 145 \\
\hline Singles $(\mathrm{kcps})^{\dagger}$ & 296 & 207 & 492 & 412 & 536 & 195 & 232 \\
\hline NECR $(k=1)(c p s)$ & 1,474 & 793 & 154 & 145 & 107 & 400 & 503 \\
\hline NECR $(k=2)$ (cps) & 1,247 & 668 & 94 & 89 & 61 & 323 & 401 \\
\hline Scatter fraction $(\%)$ & 30 & 21 & 15 & 10 & 17 & 25 & 25 \\
\hline Breast volume in PET FOV $\left(\mathrm{cm}^{3}\right)$ & 1,077 & 532 & 204 & 185 & 391 & 965 & 1,001 \\
\hline
\end{tabular}




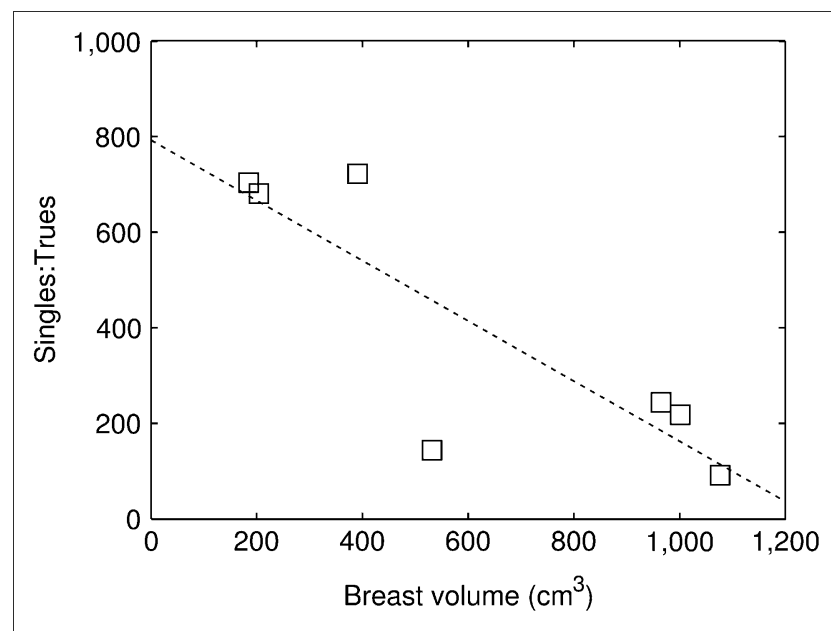

FIGURE 6. Plot of STR vs. breast volume in PET FOV for patient scans. Data were fitted with first-order polynomial $(-)$, with correlation coefficient $\left(R^{2}\right)$ of 0.72 .

whether dedicated breast PET/CT has a useful role in the clinical management of patients with primary breast cancer.

\section{ACKNOWLEDGMENTS}

We thank Nikki Emerson, Sheila Rejano, Ken Eldridge, Tina Luthge, Tashina Hayduk, Naomi Miyao, and John Brock for assistance with patient scans. This work was funded in part by the California Breast Cancer Research Program (grant 11IB-0114), the American Cancer Society (grant IRG-95-125-07), and the Susan G. Komen for the Cure foundation (grant BCT40707455). John M. Boone is a paid consultant of Varian Imaging Products, and receives research funding from Varian Imaging Systems, Hologic, Inc., and Fuji Medical Systems. Ramsey D. Badawi is a paid consultant for Toshiba Medical Research Unit.

\section{REFERENCES}

1. Rousseau C, Devillers A, Sagan C, et al. Monitoring of early response to neoadjuvant chemotherapy in stage II and III breast cancer by [F-18]fluorodeoxyglucose positron emission tomography. J Clin Oncol. 2006;24:5366-5372.

2. Mahner S, Schirrmacher S, Brenner W, et al. Comparison between positron emission tomography using 2-[fluorine-18]fluoro-2-deoxy-D-glucose, conventional imaging and computed tomography for staging of breast cancer. Ann Oncol. 2008;19:1249-1254.

3. Blodgett TM, Meltzer CC, Townsend DW. PET/CT: form and function. Radiology. 2007;242:360-385.

4. Tatsumi M, Cohade C, Mourtzikos K, Fishman EK, Wahl RL. Initial experience with FDG-PET/CT in the evaluation of breast cancer. Eur J Nucl Med Mol Imaging. 2006;33:254-262.

5. Radan L, Ben-Haim S, Bar-Shalom R, Guralnik L, Israel O. The role of FDGPET/CT in suspected recurrence of breast cancer. Cancer. 2006;107:2545-2551.

6. Avril N, Rose CA, Schelling M, et al. Breast imaging with positron emission tomography and fluorine-18 fluorodeoxyglucose: use and limitations. J Clin Oncol. 2000;18:3495-3502.

7. Kumar R, Chauhan A, Zhuang HM, Chandra P, Schnall M, Alavi A. Clinicopathologic factors associated with false negative FDG-PET in primary breast cancer. Breast Cancer Res Treat. 2006;98:267-274.

8. Rosen EL, Turkington TG, Soo MS, Baker JA, Coleman RE. Detection of primary breast carcinoma with a dedicated, large-field-of-view FDG PET mammography device: initial experience. Radiology. 2005;234:527-534.
9. Abreu MC, Aguiar JD, Almeida FG, et al. Design and evaluation of the Clear-PEM scanner for positron emission mammography. IEEE Trans Nucl Sci. 2006;53:71-77.

10. Murthy K, Aznar M, Thompson CJ, Loutfi A, Lisbona R, Gagnon JH. Results of preliminary clinical trials of the positron emission mammography system PEM-I: a dedicated breast imaging system producing glucose metabolic images using FDG. J Nucl Med. 2000;41:1851-1858.

11. Freifelder R, Cardi C, Grigoras I, Saffer JR, Karp JS. First results of a dedicated breast PET imager, BPET, using $\mathrm{NaI}(\mathrm{Tl})$ curve plate detectors. IEEE Nucl Sci Symp Conf Rec. 2001;3:1241-1245.

12. Berg WA, Weinberg IN, Narayanan D, et al. High-resolution fluorodeoxyglucose positron emission tomography with compression ("positron emission mammography") is highly accurate in depicting primary breast cancer. Breast $J$. 2006;12:309-323.

13. Orlov SS. Theory of 3-dimensional reconstruction.1. Conditions for a full set of projections. Kristallografiya. 1975;20:511-515.

14. Raylman RR, Majewski S, Smith MF, et al. The positron emission mammography/ tomography breast imaging and biopsy system (PEM/PET): design, construction and phantom-based measurements. Phys Med Biol. 2008;53:637-653.

15. Wu Y, Bowen SL, Yang K, et al. PET characteristics of a dedicated breast PET/ CT scanner prototype. Phys Med Biol. 2009;54:4273-4287.

16. Wang GC, Huber JS, Moses WW, Qi J, Choong WS. Characterization of the LBNL PEM camera. IEEE Trans Nucl Sci. 2006;53:1129-1135.

17. Tai YC, Wu H, Pal D, O'Sullivan JA. Virtual-pinhole PET. J Nucl Med. 2008;49:471-479.

18. Li HD, Wong WH, Baghaei $\mathrm{H}$, et al. The engineering and initial results of a transformable low-cost high-resolution PET camera. IEEE Trans Nucl Sci. 2007;54:1583-1588.

19. Kwan ALC, Boone JM, Yang K, Huang SY. Evaluation of the spatial resolution characteristics of a cone-beam breast CT scanner. Med Phys. 2007;34:275-281.

20. Lindfors KK, Boone JM, Nelson TR, Yang K, Kwan ALC, Miller DF. Dedicated breast CT: initial clinical experience. Radiology. 2008;246:725-733.

21. Doshi NK, Shao YP, Silverman RW, Cherry SR. Design and evaluation of an LSO PET detector for breast cancer imaging. Med Phys. 2000;27:1535-1543.

22. Boone JM, Shah N, Nelson TR. A comprehensive analysis of $\mathrm{DgN}(\mathrm{CT})$ coefficients for pendant-geometry cone-beam breast computed tomography. Med Phys. 2004;31:226-235.

23. Boone JM, Kwan ALC, Seibert JA, Shah N, Lindfors KK, Nelson TR. Technique factors and their relationship to radiation dose in pendant geometry breast CT. Med Phys. 2005;32:3767-3776.

24. Qi J, Leahy RM, Cherry SR, Chatziioannou A, Farquhar TH. High-resolution 3D Bayesian image reconstruction using the microPET small-animal scanner. Phys Med Biol. 1998;43:1001-1013.

25. Feldkamp LA, Davis LC, Kress JW. Practical cone-beam algorithm. J Opt Soc Am A Opt Image Sci Vis. 1984;1:612-619.

26. Studholme C, Hill DLG, Hawkes DJ. An overlap invariant entropy measure of 3D medical image alignment. Pattern Recognit. 1999;32:71-86.

27. Rorden C, Brett M. Stereotaxic display of brain lesions. Behav Neurol. 2000;12:191-200.

28. American College of Radiology. Breast Imaging Reporting and Data System (BI-RADS): Mammography. 4th ed. Reston, VA: American College of Radiology; 2003.

29. Strother SC, Casey ME, Hoffman EJ. Measuring PET scanner sensitivity: relating countrates to image signal-to-noise ratios using noise equivalent counts. IEEE Trans Nucl Sci. 1990;37:783-788.

30. Watson CC, Casey ME, Bendriem B, et al. Optimizing injected dose in clinical PET by accurately modeling the counting-rate response functions specific to individual patient scans. $J$ Nucl Med. 2005;46:1825-1834.

31. Jan S, Santin G, Strul D, et al. GATE: a simulation toolkit for PET and SPECT. Phys Med Biol. 2004;49:4543-4561.

32. Qi J, Huesman RH. Scatter correction for positron emission mammography. Phys Med Biol. 2002;47:2759-2771.

33. Miglioretti DL, Rutter CM, Geller BM, et al. Effect of breast augmentation on the accuracy of mammography and cancer characteristics. JAMA. 2004;291:442-450.

34. Mawlawi O, Podoloff DA, Kohlmyer S, et al. Performance characteristics of a newly developed PET/CT scanner using NEMA standards in 2D and 3D modes. J Nucl Med. 2004;45:1734-1742.

35. McCollough $\mathrm{CH}$, Zink FE. Performance evaluation of a multi-slice CT system. Med Phys. 1999;26:2223-2230.

36. Zhang J, Olcott PD, Chinn G, Foudray AMK, Levin CS. Study of the performance of a novel $1 \mathrm{~mm}$ resolution dual-panel PET camera design dedicated to breast cancer imaging using Monte Carlo simulation. Med Phys. 2007;34:689-702.

37. Boone JM, Kwan ALC, Yang K, Burkett GW, Lindfors KK, Nelson TR. Computed tomography for imaging the breast. J Mammary Gland Biol Neoplasia. 2006;11:103-111. 